\title{
Cellular Signaling Pathways and Vascular Dysfunctions
}

\author{
Gundu HR Rao* \\ Laboratory Medicine Pathology Director, Thrombosis Research, Lillehei Heart Institute, University of Minnesota, USA
}

Submission: November 01, 2018; Published: December 07, 2018

*Corresponding author: Gundu HR Rao, Laboratory Medicine Pathology Director, Thrombosis Research, Lillehei Heart Institute, University of Minnesota, 12500 Park Potomac Ave, Unit 306N Potomac, MD 20854, USA

\begin{abstract}
Metabolic diseases such as hypertension, excess weight, obesity, endothelial dysfunction, type-diabetes and vascular diseases have reached epidemic proportions worldwide. Several clinical trials have demonstrated that robust management of modifiable risks factors will reduce premature mortality. Having said that, such a management strategy for risk modification will not reduce or reverse any of the metabolic diseases. In view of this observations, we feel strongly that diagnosis of the early risk factors or metabolic disturbances and developing novel approaches for such risks will be a better choice for the reduction of prevention of these diseases. Furthermore, we believe that ultimate clinical complications of major metabolic diseases or related to altered vascular physiology and function. Entire set of signaling pathways induced by interactions of agonists or antagonists at the receptor level is called signal transduction mechanism or pathways. The ability of cells, tissues and organs, to perceive and to respond to alterations in the microenvironment is the primary basis of physiology and function of these systems and the basis for the cell, tissue and organ repair, immunity and homeostasis. Alterations or errors in signaling process and information processing are responsible for the development of metabolic risks. In this mini review, we will briefly discuss altered metabolism during intrauterine growth, oxidative stress, low grade chronic inflammation, fatty acid metabolism, and cytosolic calcium modulation, as they relate to metabolic diseases. Progress in emerging technologies such as fluorosequencing, will enable the researchers to identify at single molecule level and enhance the detection and management of biomarkers of clinical, diagnostic and therapeutic importance.
\end{abstract}

Keywords: Vascular dysfunctions; Hypertension; Excess weight; Obesity; Endothelial dysfunction; Type-diabetes; Fatty acid metabolism, Cytosolic calcium modulation

Abbreviations: CVD: Cardiovascular Disease; HDL-C: High-Density Lipoproteins; BP: Blood Pressure; PFAA: Plasma Free Amino Acids; FOAD: Fetal Origin of Adult Disease; PKC: Protein Kinase C; NO: Nitric Oxide; PG: Prostaglandins; ENOS: Endothelial cell NO Synthase

\section{Introduction}

Metabolic diseases such as hypertension, obesity, type-2 diabetes contribute significantly to the global burden of vascular disease related mortality and morbidity. In the past three decades, these metabolic diseases have reached epidemic proportions worldwide [1-17]. No country had reduced or reversed the increase in the incidence of these metabolic diseases. However, it is to be noted that there is considerable decline in the premature mortality due to Cardiovascular Disease (CVD) in industrialized countries [8-11]. This observed decline seems to be due to a healthy lifestyle and better management of modifiable risk factors [8]. According to the experts, a constellation of metabolic abnormalities including centrally distributes obesity (Asian phenotype), decreased levels of High-Density Lipoproteins (HDL-C), elevated triglycerides, elevated Blood Pressure (BP), and hyperglycemia is known as metabolic syndrome [7]. This syndrome seems to be the driver for the modern-day epidemics of diabetes and CVD and has become a great public health challenge worldwide. Once the metabolic risk has become well characterized into one or more clusters of known risk factors, then we are left with no choice, but to manage the modifiable risks effectively, to prevent premature mortality and comorbidities associated with the progression of multiple risk factors. In view of this observation, we feel that the need of the hour is, to concentrate on the early diagnosis of metabolic alterations and develop appropriate novel interventions, to prevent the progress of these altered metabolic states.

We have articulated in many of our recent articles that the earliest altered metabolic risk that can be diagnosed and managed very well is the intrauterine alterations in the development of fetus. Having said that, we need to explain this phenomenon better as we have encountered some concerns form experts who are not familiar with this condition. In Asian countries including India and China with large population, even to this day $30 \%$ of the children born are of low birth-weight [18]. These low birth weight children are "at risk" for developing metabolic disease during their middle age. Based on the epidemiological studies in the UK and India, David Barker, a British Epidemiologist proposed a hypothesis, that intrauterine growth retardation, low birth weight, and premature birth have a relationship to the origins of hypertensions coronary 
artery disease, and noninsulin-dependent diabetes in the middle age [19]. According to researchers, the fact that intrauterine growth retardation may predispose the nutrition-related chronic disease has serious implications for developing countries, as it may add to the burden of existing obesity and diabetes epidemic, as diets and lifestyles in these countries are in themselves "atherogenic [20]." Researchers from Harvard School of Public Health have reported results of a meta-analysis, on the benefits of multiple micronutrient supplementation to improve maternal and infant health [21].

The next phase in the diagnosis of "at risk" subjects is infants under two years age. A new study from a collaborative group from Colorado School of Public Health, Norwegian School of public health, University of Oslo, and University of San Diego have reported that gut microbiota from early life to two years shows an increasingly strong association with childhood BMI. This study for the first time provides evidence that the gut microbiome at 2 years of age may offer useful information to help to identify youth who are at risk for obesity, which could facilitate more targeted early prevention efforts [22]. These researchers also found that maternal overweight/obesity may influence some of the specific gut microbiota taxa that are associated with later BMI. It is of great importance to find out as to what kind of gene expression or molecular signalizing is involved in the reprogramming of the infant's metabolism to predispose them to overweight and obesity in their later life. Childhood obesity is a global public health problem. Many genetic, epigenetic, behavioral, dietary, physical activity or the lack of it, play a role in the progress of this altered metabolic state [23]. Being underweight, overweight, or obese during childhood and adolescence is associated with adverse health consequences throughout the life of the individuals. Furthermore, being overweight in childhood and adolescence is associated with greater risk and earlier onset of chronic disorders such as type- 2 diabetes.

Japanese workers presented their findings in the recent meetings at Berlin, Germany suggesting that measuring just three risk factors, - increase in fasting glucose, insulin sensitivity and could predict the probability of developing prediabetes 10year before it occurs. Since it takes another ten years to develop diabetes, it is possible to diagnose these early biomarkers for diabetes 20 years ahead of its occurrence. In addition, studies have demonstrated that presence of Plasma Free Amino Acids (PFAA) also, is a strong predictor of future diabetes development [24-27]. To add to this complexity, it has been shown that gut microbiome can influence modulate the amino acid metabolism and induce increased of PFAA and short chain fatty acids, suggesting that there are alternate signaling pathways, that influence the metabolism of the host and are capable of reprograming the amino acid and fatty acid metabolism. Since some of the studies have demonstrated that the PFAA profile can also detect the development of insulin resistance, there exists signaling pathways, that can modulate both carbohydrate and amino acid metabolism. Japanese researchers have demonstrated that PFAA profiles can predict the onset of CVD in patients with type- 2 diabetes over a 10 -year follow-up period [25]. These studies underscore the potential key role of amino acid metabolism early in the pathogenesis of diabetes.

Excess weight, obesity and diabetes are usually accompanied by oxidative stress and low -grade inflammation [28,29]. Inflammation, endothelial dysfunction, and hypercoagulabity are correlated to each other, thus playing an important role in the development of vascular complications in diabetes patients. Development of these altered metabolic state could be monitored aby assaying the biomarkers such as VWF, IL-6, TNF-alpha, D-dimer and PAI-1 [28]. Any imbalance between the reactive oxygen species and antioxidants leads to the development of oxidative stress [29]. Free radicals are important for activating different signaling pathways in the cell, such as various kinases that alter gene expression as well as coordination with superoxide dismutase. Kelly and associates studied the role of exercise in modulating the inflammation, insulin, and endothelial dysfunction in overweight children and adolescents [30]. In overweight children and adolescents, they found that C-reactive protein was independently associate with fasting insulin. Just eight weeks of aerobic exercises seem to improve fitness, HDL-cholesterol, and endothelial function in this group.

A brief review of literature shows that altered molecular and cellular signaling pathways could alter metabolic state right at the intrauterine growth level, to infants, young children, adolescents and prediabetics. If we understand the mechanisms involved in these signaling pathways, then we can develop appropriate interventions to modulate the signaling pathways to prevent or reduce the metabolic risks that promote metabolic diseases and contribute to the premature mortality and comorbidities. In this mini review we will discuss, some signaling pathways as examples of early diagnosis, management of observed risks, and present over views on possible development of complementary therapies.

\section{Discussion}

The theme of this mini review was cellular and molecular signaling pathways and their role in modulating vascular function. We have used the word vascular dysfunction to denote both hypofunction and hyperfunction. We have articulated in our earlier articles that early diagnosis risk factors and effective management of observed risks will prevent premature mortality and comorbidities [31-36]. Several studies from India, China and the UK have reported that intrauterine growth disturbances and resulting low-weight at birth predisposes children to various metabolic diseases at their middle age [18]. Based on these early studies Dr. David Barker, the British epidemiologist developed his hypothesis, that intrauterine growth retardation, low-birth weight, and premature birth have a causal relationship to the origins of hypertension, coronary heart disease, and noninsulindependent diabetes, in middle age. Fetal Origin of Adult Disease (FOAD) is associated with low-birth weight, a surrogate marker for poor fetal growth and nutrition, is linked to coronary artery disease, hypertension, obesity and insulin resistance [18-23]. Earlier studies on this problem focused heavily on the fetal programing of nutrition-related chronic diseases. Recent studies 
are exploring other alternate molecular mechanisms, such as miRNA or microbiome mediated signaling pathways in the fetal programing for chronic adult metabolic studies [22,37].

\section{Intrauterine Signaling Pathways}

It is believed that adipocytes in obese individuals induce systemic inflammation via secreted cytokines, however, recent studies from the Children's Hospital, Washington DC has explored another potential mediator which has been identified as adipocyte-derived exosomes rich in miRNA [37]. Based on these observations, a bilateral research project funded by the National Institutes of Health (NIH, USA) has been developed between the staff of Children's Hospital, USA and Diabetes Clinic staff of KEM Hospital, Pune, India. In view of the earlier observations and the importance of the visceral adipose depot in obesity-related comorbidities, such as cardiovascular disease and metabolic syndrome, a comparative study has been initiated to look at the miRNA- mediated gene expression in obese vs lean individuals, and the influence of exosomes shed by obese pregnant women vs lean pregnant women on their off springs' growth and development. Preliminary studies are encouraging, and further exploration of this pathways are important, as infant and childhood obesity may result in early and cumulative epigenetic changes leading to obesity-related metabolic diseases.

\section{Oxidative Stress and Inflammation Pathways}

Metabolic disorders by and large, lead to oxidative stress followed production of oxygen species (ROS) and injury of cellular and molecular pathways by the action of ROS. It also affects insulin sensitivity and activity through several interacting pathways. The ROS can also damage the B-cells the pancreas, resulting reduced production of insulin. Furthermore, these mechanisms could affect NF-kB nuclear factor-kB) and Protein Kinase C (PKC) pathways and interfere with insulin signaling pathways [38-41]. Inflammation seems to be the major initiator of many processes that leads to self-healing as well as pathogenesis of major metabolic diseases, including, atherosclerosis, obesity, metabolic syndrome, type-2 diabetes, ischemic heart disease and stroke. Inflammation seems to play a role even in acute events such as vulnerable plaque rupture, precipitation of some of the events associated with stroke, advance events in cancer and neuronal pathology. The inflammatory condition on the other hand, triggers the development of insulin resistance and diabetes through a complex pathway, consisting of several kinases and signaling pathways [41]. The adipocytes and immunocytes produce various proinflammatory cytokines including IL-6, TNF-alpha, that are involved in the pathogenesis of diabetes.

The transcription factor Nrf2 (nuclear factor erythroid2-reoated factor-2, Nrf-2) for instance, a master regulator of detoxification, anti-oxidant, anti-inflammatory and other cytoprotective mechanisms, is raised by health promoting factors. This transcription factor activates the transcription of over 500 genes (so called survival genes) in the human genome, most of which have cytoprotective functions. The most healthful diets such as Mediterranean and Okinawa are rich in Nrf2 raising nutrients. the levels of Nrf2 levels. Recent studies, however, have demonstrated that induction of Nrf2 and Ho-1 expression by Protandim (a mixture of five phytochemicals; Aashaadha, Indian Bacopa, Indian Green Tea, China Milk Thistle and China Turmeric) is associated with a reduction in oxidative stress and fibrosis, preservation of the RV microcirculation and RV function [8]. Studies by Joe M McCord and associates on the effect of Protandim on various pathways have shown, significant modulation by Protandim not only of pathways involving antioxidant enzymes, but also those related to Colon Cancer, Cardiovascular disease and Alzheimer's disease [42,43]. On the other hand, Beijing University researchers have reported that an herbal product Berberine, can combat both oxidative stress as well as inflammation and thus ameliorate diabetes [41]. Although the researchers speculate that it works on Nff2 and AMP kinases, they acknowledge the need for additional studies to explain the molecular mechanisms involved in the beneficial effects of Berberine.

\section{Endothelial Dysfunction Pathways}

As mentioned in several of our recent articles, the early metabolic risks include, intrauterine disturbances, oxidative stress, low-grade chronic inflammation and endothelial dysfunction. One of the pioneer cardiologists, Professor Jay Cohn says, that there is no vascular disease if there is no endothelial dysfunction. Vascular endothelium is the major source of overprotective molecules such as adenosine, prostacyclin, prostaglandin E1, and Nitric Oxide (NO), -the most potent vasodilator [44]. When the balance between the vasoconstrictors and vasodilators are altered endothelial dysfunction occurs, causing damage to the arterial wall. Endothelial dysfunction is considered the earliest marker for atherosclerosis, preceding angiographic or ultrasonic evidence of atherosclerotic plaque. Studies from our laboratory at the University of Minnesota, demonstrated that in a drug-induced diabetes model the arachidonic acid metabolism is altered in favor of excess production of pro aggregatory prostaglandins (PG) $\mathrm{PGG}_{2}$ and $\mathrm{PGH}_{2}$ ad Thromboxane $\mathrm{A}_{2}$. The altered balance also shifted towards lowered production of vasodilatory prostacyclins. We were able to bring back the normal state of arachidonic acid metabolism aby the transplant of islet cells and modulating the glycemic state [45]. The maintenance of vascular tone seems to be accomplished by the release of various vasodilator and vasoconstrictor molecules. During oxidative stress and inflammation, endothelial cells undergo a phenotypic conversion, resulting in the activation of the endothelial cell, characterized by enhanced cell permeability, elevated leukocyte adhesion molecule expression, and reduced anti-thrombotic properties. Endothelial dysfunction results from a decrease in the bioavailability of nitric oxide, a crucial regulator of vascular tone [46]. Variety of stimuli, including shear stress, acetylcholine, bradykinin, insulin, and adiponectin activate endothelial cell NO synthase (eNOS) to convert L-arginine to citrulline, substrates for NO production. It I believed that effect of hyperglycemia on endothelial cells closely resemble that of inflammatory mediators. Although we did not 


\section{Journal of Cardiology \& Cardiovascular Therapy}

measure No production in our drug-induced diabetes model, the imbalance that we observed for AA pathway also is paralleled by imbalance in No production, which is normalized by the restoration of normal glycemia (Figure 1).

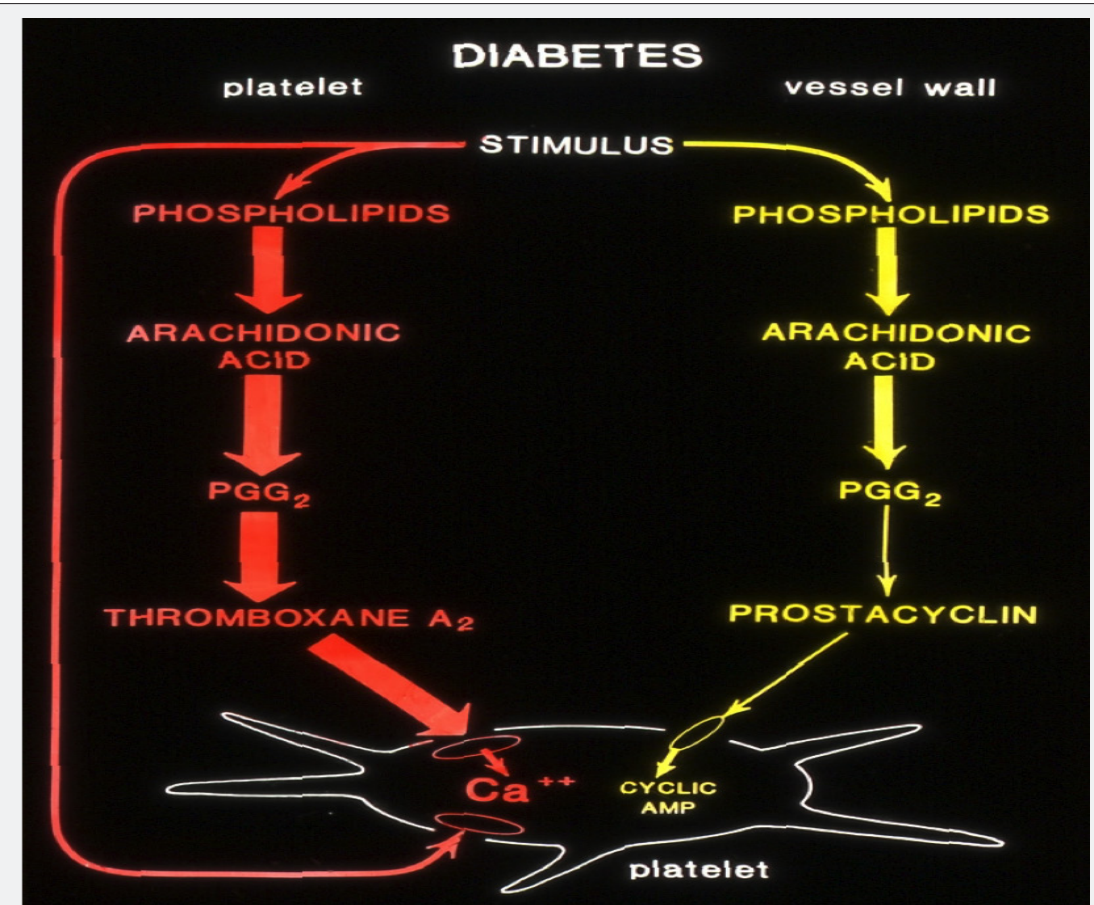

Figure 1: Altered Prostaglandin and Prostacyclin generation in drug-induce diabetes.

A number of interventions have been shown to be effective in restoring endothelium-dependent vasodilation. These include lipid-loweringtherapy (e.g., 3-hydroxy-3-methylglutaryl coenzyme A [HMG-CoA] reductase inhibitors [statins], cholestyramine, or LDL apheresis), angiotensin-converting enzyme inhibitors, antioxidants, reducing hyperglycaemia, diet, and exercise. The effectiveness of dietary L-arginine on endothelial dysfunction in humans is not fully established and further research is needed. In a preliminary study we were able to demonstrate.

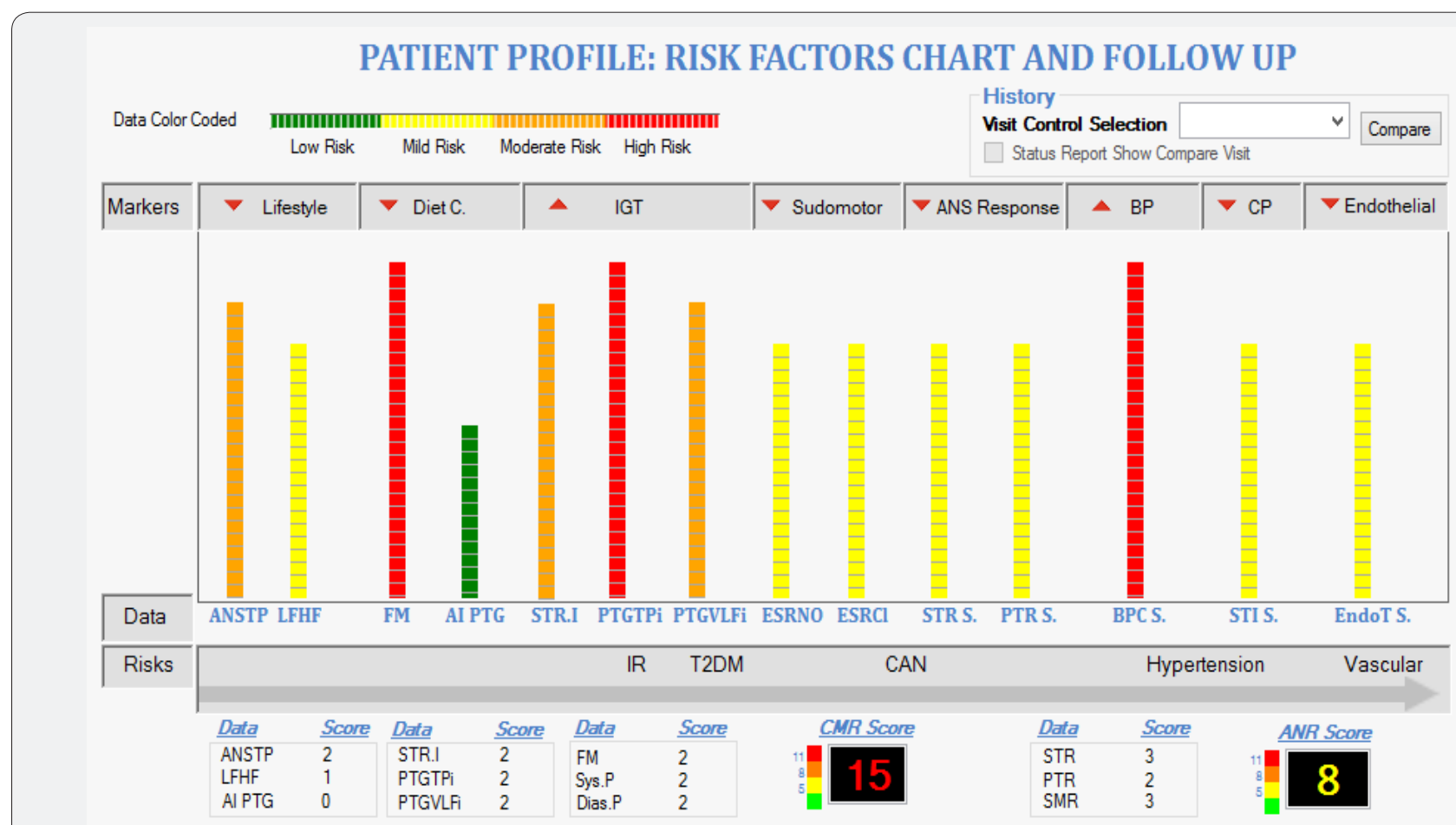

Figure 2: Risk Factor profile of a patient with TM-Oxi system (LD-Technologies). 
The restoration of endothelial dysfunction in a patient by restoring normoglycemia and dietary supplement of L-arginine. Figure 2 shows the profile of a patient data before the recommendation of lifestyle changes and dietary supplementation of L-arginine. Note the cardiometabolic score color coded in red with a score of 15. Shown in the (Figure 3). Are values.

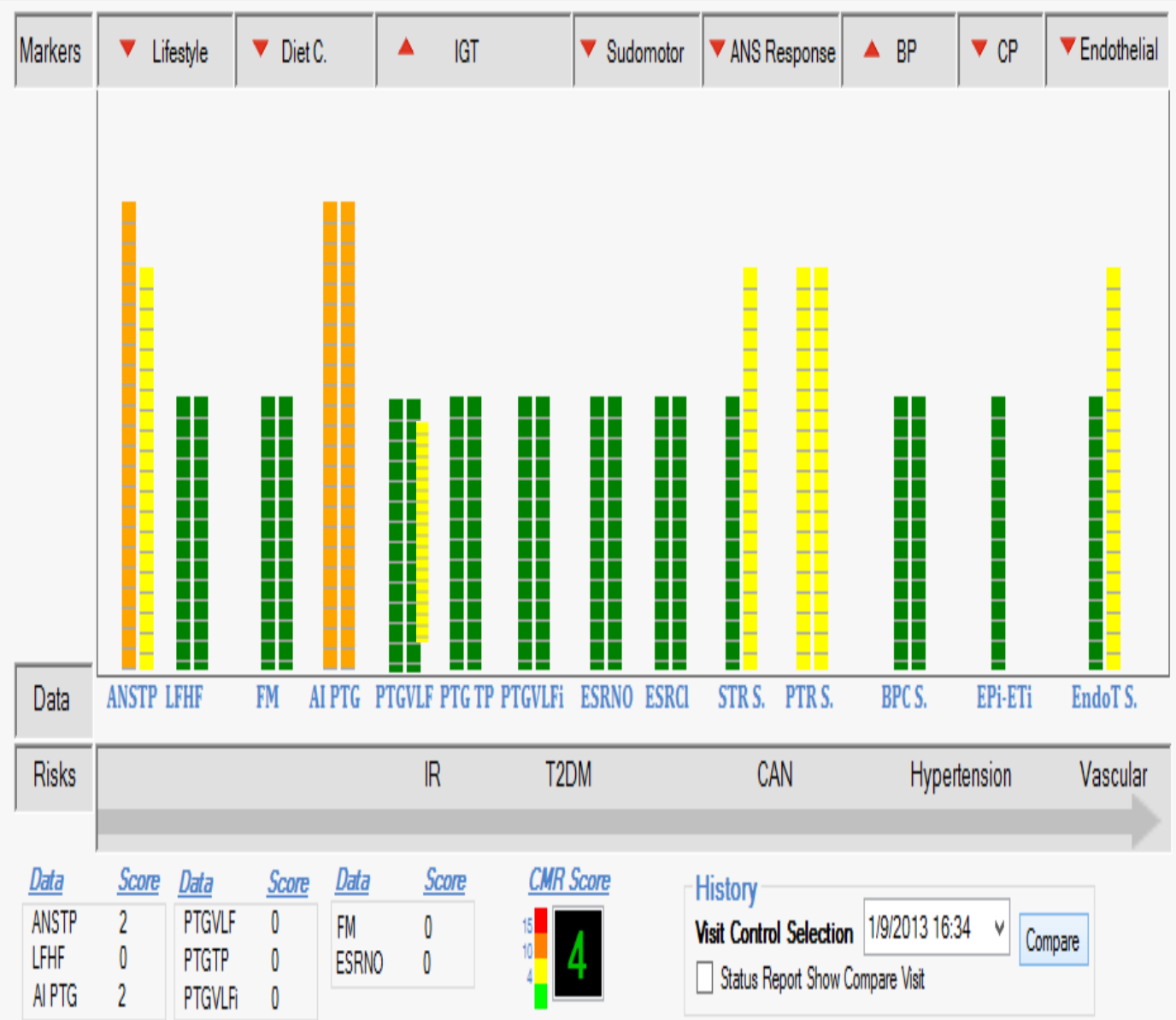

Figure 3: Risk Factor Profile of the patient post-treatment and Lifestyle changes. LD-technologies, Miami, Florida. (Courtesy: Dr. Gandhi, $P$ and Dr. Maarek A).

After 8 weeks of treatment, which included dietary changes, increased physical activity, metformin $500 \mathrm{mg}$ two tablets twice daily) and L-arginine tablets twice daily). Note the drastic reduction in the CMR score post treatment (CMR score is a composite of several risk monitored: vascular risk factors, autonomous nervous system tests, and hemodynamic tests).

\section{Conclusion}

Theability of cells, tissues and organs, toperceiveand torespond to alterations in the microenvironment is the primary basis of physiology and function of these systems and the basis for the cell, tissue and organ repair, immunity and homeostasis. Alterations or errors in signaling process and information processing are responsible for the development of metabolic risks. In this mini review, we have tried briefly to discuss altered metabolism during intrauterine growth, oxidative stress, low grade chronic inflammation, fatty acid metabolism, and endothelial dysfunction as they relate to the development of metabolic diseases. Several major clinical trials have demonstrated the beneficial effect of a healthy diet and life style on the observed decline in CVD-related premature mortality and associated comorbidities. However, when an obese or diabetic patient walk into a clinic, the choice of treatments is limited. In majority of clinics, the first line of treatment protocol would be to treat observed hypertension, lipid abnormalities and hyperglycemia. The main goals would be to normalize the blood pressure, blood lipids and blood glucose. We have shown that in experimental studies with short-term goals, one can achieve normalization. However, the big question seems to be what does this mean to the various pathological changes that have already occurred? In view of the fact that there are no easy ways to measure associated clinical complications, clinicians have limited their treatment protocols to manage observed risk factors and assure the patients that everything is $\mathrm{OK}$.

With the use of emerging technologies, it is possible to monitor a variety of metabolic risks, develop complimentary therapies to observed risks (oxidative stress, inflammation, endothelial dysfunction etc.), and follow the effect of such therapies, to find out the outcome of such approaches. In our next article in this journal, we will elaborate on this topic, and discuss the effectiveness or otherwise of management of diabetes and cardiometabolic diseases, using a novel integrated diagnostic platform. 


\section{Journal of Cardiology \& Cardiovascular Therapy}

\section{References}

1. Forouzanfar M, Liu P, Roth GA, Ng M, Biryukov S, et al. (2017) Global burden of hypertension and systolic blood pressure of at least 110 to 115 mm Hg, 1990-2015. JAMA 317(2): 165-182.

2. Van Gaal LF, Mertens IL, De Block CE (2006) Mechanisms linking obesity with cardiovascular disease. Nature 444(7121): 875-880.

3. GBD 2015 Risk Factors Collaborators (2015) Global, regional, and national comparative risk assessment of 79 behavioral, environmental and occupational, and metabolic risk or clusters of risks, 1990-2015: a systematic analysis for the Global Burden of Diseases Study. Lancet. 388(10053): 1659-1724.

4. Zheng X, Jin C, Liu Y, Zhang J, Zhu Y, et al. (2015) Arterial stiffness as a predictor of clinical hypertension. J Clin Hyperten (Greenwich) 17(8): 582-291.

5. Ng M, Fleming T, Robinson M, Thomson B, Graetz N, et al. (2013) Global, regional, and national prevalence of overweight and obesity in children and adults during 1980-2013: a systematic analysis for the Global Disease Study. Lancet 384(9945): 766-781.

6. Lu Y, Hajifathalian K, Ezzati M, Woodward M, Rimm EB, et al: (2014) Global Burden of Metabolic mediators of the Risk Factors for Chronic Diseases Collaborations (BMI Mediated Effects). Metabolic mediators of the effect of body-mass index, overweight and obesity on coronary heart disease and stroke: a systematic analysis of 97 prospective cohorts with 1.8 million participants. Lancet 383(9921): 970-983.

7. Zimmet P, Magliano D, Matsuzawa Y, Alberti G, Shaw J, et al. (2005) The metabolic syndrome: a global public health problem and a new definition. J Atheroscler Thromb 12(6): 295-300

8. Yusuf S, Hawken S, Ounpuu S, Dans T, Avezum A, et al. (2004) Effect of modifiable risk factors associated with myocardial infarction in 52 countries (the INTERHEART study): case-control study. Lancet 364(9438): 937-952.

9. Taylor R, Dobson A, Mirzaei M (2006) Contribution of changes in risk factors to the decline of coronary heart disease mortality in Australia over three decades. Eur J Cardiovasc Prev Rehabil 13(5): 760-768.

10. Luepker RV (2008) Decline in incident of coronary heart disease: why are the rates falling? Circ 117(5): 592-593.

11. Hata J, Ninomiya T, Hirakawa Y, Nagata M, Mukai N, et al. (2013) Secular trends in cardiovascular disease and its risk factors in Japanese: half century data from Hisayama Study (1961-2009). Circ 128(11): 11981205.

12. Krug EG (2016) Trends in diabetes: sounding the alarm. Lancet 387(10027): 1485-1486.

13. Smith-Sprangler CM, Bhattacharya J, Goldhaber-Fiebert JD (2012) Diabetes, its treatment and catastrophic medical spending in 35 developing countries. Diabetes Care 35(2): 319-326.

14. NDC Risk Factor Collaboration (NCD-RisC) (2016) Worldwide trends in diabetes since 1980: a pooled analysis of 751 population-based studies with 4.4 million participants. Lancet 387(10027): 1513-1530.

15. Merlotti C, Morabito A, Pontiroli AE (2014) Prevention of type2 diabetes; a systematic review and meta-analysis of different intervention strategies. Diabetes Obes Metab 16(8): 719-727.

16. Li G, Zhang P, Wang J, Gregg EW, Yang W, et al (2008) The long-term effect of lifestyle interventions to prevent diabetes in the China Da Qing Diabetes Prevention Study: a 20year follow-up study. Lancet 371(9626): 1783-1789.

17. Knowler WC, Barrett-Connor E, Fowler SE, Hamman RF, Lachin JM, et al (2002) Reduction in the incidence of type-2 diabetes with lifestyle intervention or metformin. N Engl J Med 346: 393-403.

18. Rao GHR, Gandhi PG, Sharma V (2014) Clinical complications of type- 2 diabetes mellitus in South Asian and Chinese populations: An overview. J Diabetes Metab 5: 420.

19. Calkins K, Devaskar SU (2011) Fetal origin of adult disease. Curr Probl Pediatr Adolesc Health Care 41(6): 158-176.

20. Delisle H (2002) Foetal programming of nutrition-related chronic diseases. (article in French). Sante 12(1): 56-63.

21. Kawai K, Spiegelman D, Shankar AH, Fawzi WW (2011) Maternal multiple micronutrient supplementation and pregnancy outcomes in developing countries: meta-analysis and meta-regression. Bull World Health Organ 89(6): 402-411.

22. Stansilawski MA, Dabelea D, Wagner BD et al 0 Gut microbiota in the first 2 years of life and the association with Body mass index at age 12 in a Norwegian birth cohort. Am Soc. Microbiol. mBio. 9e01751-18. https://doi.org/10.1128/mBio.01751-18

23. NCD Risk Factor Collaboration (NCD-RisC) (2017) Worldwide trends in body-mass index, underweight, and obesity from 1975 to 2016: a pooled analysis of 2416 population-based studies in 128.9 million children, adolescents and adults. Lancet 390(10113): 2627-2642.

24. Wurtz P, Soininen P, Kangas AJ, Rönnemaa T, Lehtimäki T, et al. (2013) Branched-chain aromatic amino acids are predictors of insulin resistance in young adults. Diabetes Care 36(3): 648-655.

25. Kume S, Araki S, Ono N, Shinhara A, Muramatsu T, et al. (2014) Predictive properties of plasma free amino acid profile for cardiovascular disease in patients with type-2 diabetes. PLOS One 9(6): e10219.

26. Wang TJ, Larson MG, Vasan RS, Cheng S, Rhee EP, et al (2011) Metabolite profiles and the risk of developing diabetes. Nat Med 17(4): 448-453.

27. Magnusson M, Lewis GD, Ericson U, Orho-Melander M, Hedblad B, et al. (2013) A diabetes-predictive amino acid score and future cardiovascular disease. Eur J Heart J 34(26): 1982-1989.

28. Domingueti CP, Dusse LM, Carvalho Md, de Sousa LP, Gomes KB, et al. (2016) Diabetes mellitus: The linkage between oxidative stress, inflammation, hypercoagulabity, and vascular complications. J Diab Compl 30(4): 738-745.

29. Asmat U, Abad K, Ismail K (2016) Diabetes mellitus and oxidative stress- A concise review. Saudi Pharm J 24(5): 547-553.

30. Kelly AS, Wetzsteon RJ, Kaiser DR, Steinberger J, Bank AJ, et al. (2004) Inflammation, insulin, and endothelial function in overweight children and adolescents: the role of exercise. J Peadiatr 145(6): 731-736.

31. Rao GHR (2018) Prevention or reversal of cardiometabolic diseases. J Clin Prevent Cardiol 7(1): 22-28.

32. Rao GHR (2018) Importance of studying disease and disease process. Editorial. Developments in Clin Med Pathol Crimson Publ 1: 1.

33. Rao GHR (2018) Cellular and Molecular Approaches to Therapy. Clin Med Pathology 1(3): DCMP.000511.

34. Rao GHR (2018) Reduction reversal or prevention of type-2 diabetes mellitus. Editorial. Arch. Endocrinology and Diabetes Care 1(1): ADO. MS.ID.000101.

35. Rao GHR (2018) Cardiometabolic Diseases: A global perspective. J of Cardiol Cardiovasc Ther 12(2): JOCCT.MSID.555834.

36. Rao GHR (2018) Diagnosis of early risks, management of risks, and reduction of vascular diseases. J Clin Card \& Diagnostics 1(1): 1-11.

37. Ferrante SC, Nadler EP, Pillai DK, Hubal MJ, Wang Z, et al. (2015) Adipocyte-derived exosomal miRNA: a novel mechanism for obesityrelated disease. Pediatr Res 77(3): 447-454.

38. BabizhaYev MA, Strokov IA, Nosikov VV, Savel'yeva EL, Sitnikov VF, et al. (2015) The role of oxidative stress in diabetic neuropathy: Generation of free radical species in the glycation reaction and gene polymorphisms encoding antioxidant enzymes to genetic susceptibility to diabetic neuropathy in population of type- 1 diabetic patients. Cell Biochem Biophys 71(3): 1425-1443. 
39. Osawa T, Kato Y (2005) Protective role of antioxidative food factors in oxidative stress cause by hyperglycemia. Ann N Y Acad Sci 1043: 440451

40. Fiorentino TV, Prioletta A, Zuo P, Folli F (2013) Hyperglycemia-induced oxidative stress and its role in diabetes mellitus related cardiovascular diseases. Curr Pharm Des 19(32): 5693-5703.

41. Ma X, Chen Z, Wang L, Wang G, Wang Z, et al. (2018) The pathogenesis of diabetes mellitus by oxidative stress and inflammation: its inhibition by Berberine. Front Pharmacol 9: 782.

42. Hybertson BM, Gao B, Bose SK, McCord JM (2011) Oxidative stress in health and disease: The therapeutic potential of Nrf2 activation. Mol Aspects Med 32(4-6): 234-246.
43. McCord JM, Fridovich I (1978) The biology and pathology of oxygen radicals. Ann Intern Med 89(1): 122-127.

44. Davignon J, Ganz P (2004) Role of endothelial dysfunction in atherosclerosis. Circulation 109(23 Suppl 1): III27-32.

45. Gerrard JM, Stuart MJ, Rao GH, Steffes MW, Mauer SM, et al. (1980) Alteration in the balance of prostaglandin synthesis in diabetic rats. J Lab Clin Med 95(6): 950-958.

46. Funk SD, Yurdagul A, Orr AW (2012) Hypoglycemia and endothelial dysfunction in atherosclerosis: Lessons from Type-1 diabetes. Int J Vasc Med 2012: 569654

Your next submission with Juniper Publishers
will reach you the below assets
- Quality Editorial service
- Swift Peer Review
- Reprints availability
- E-prints Service
- Manuscript Podcast for convenient understanding
- Global attainment for your research
- Manuscript accessibility in different formats
( Pdf, E-pub, Full Text, Audio)
- Unceasing customer service
Track the below URL for one-step submission
https://juniperpublishers.com/online-submission.php

